\title{
The Co-Evolution of Trust and Coordination in Global Software Development Teams-An Extensible Evolutionary Game Theory Model
}

\author{
Yi Wang \\ Department of Software Engineering \\ Rochester Institute of Technology \\ yi.wang@ rit.edu
}

\author{
Zhendong Wang \\ Department of Informatics \\ University of California, Irvine \\ zhendow@uci.edu
}

\author{
David Redmiles \\ Department of Informatics \\ University of California, Irvine \\ redmiles@ics.uci.edu
}

\begin{abstract}
Trust is important for effective coordination in global software development teams. However, the co-evolution of trust and coordination is often neglected. To fill the gap, we develop an evolutionary game theory model. Using the Behavior-Preference-Constraint (BPC) model and Adaptive Play, the model challenges the traditional view of trust as a static "resource" for coordination and proposes an alternative view that trust dynamically restricts people's action choices in interacting with other team members. Through analyzing the model, we describe how trust and coordination co-evolve in the progress of interactions among team members. We propose three propositions summarizing the long-term characteristics of coordination and trust in the process. For example, the co-existence of low trust and high trust can be a stable state in the long run, which explains why low trust can always exist even when all team members strictly prefer effective coordination.
\end{abstract}

\section{Introduction}

Trust is crucial for collaboration activities in global software development (GSD) teams [1, 2, 3]. Trust enables teamwork and is closely related to effective coordination $^{1}$, thereby influences team performance [6]. Researchers in many fields (e.g., CSCW, SE, IS, etc.) have been investigating trust in distributed setting extensively. Using various empirical methods, they have built solid knowledge on the antecedents of trust in GSD teams, e.g., [7, 8, 9]. We become more curious about the consequences of the trust, i.e., how trust impacts the collaborations in GSD teams. Particularly, we are interested in the co-evolution of trust and coordination for coordination is a central construct which directly influences other important factors such as team performance, conflicts, product quality $[10,11]$.

Although trust research is diverse, it is fair to say that trust is almost always viewed as a static "resource" for

\footnotetext{
1 "Coordination" means many things [4] in different context. In this paper, we follow the tradition in GSD literature [5], which restricts it to "coordinating on work."
}

interpersonal coordination, e.g., [12, 13, 14]. However, this assumption often fails to hold. People often update their trust and thereby adapt their behaviors in interactions [15]. For example, [1] suggests trust is a dynamic process interrelated with interaction outcome. The knowledge on the dynamic relationship between trust and coordination is very limited. There are many unanswered questions. E.g., how would trust and coordination co-evolve in a large enough team? Would higher trust always bring better coordination? How about the possibility of coordination failure in the long run? Is it possible to eliminate low trust using successful coordination as incentives? In essence, we want to address the following questions:

In a GSD team, how do trust and coordination dynamically co-evolve? Can we build an analytical model to describe the co-evolution?

To answer these questions, we develop an alternative view of trust based on Belief-Preference-Constraint $(B P C)$ model [16]. That is: trust is dynamic constraints of GSD team members' behaviors in interactions. Trust impact individuals' action choices, and thereby the effectiveness of coordination. Using Adaptive Play in Evolutionary Game Theory (EGT) as the analytic tool $[17,18]$, we model the long-term co-evolution of trust and coordination. Our analyses yield three main propositions. They explain why it is so hard to eliminate low trust even when all members desire and achieve effective coordination. I.e., even when some members' trust is low, a team still can achieve effective coordination because people can learn to form conventions with them. Thus, regarding observable behaviors, a team can establish "trust" in form of conventions [19]. Specifically, this work makes the following contributions:

- An alternative view of trust-Trust is conceptualized as a type of belief which enforces behavioral constraints and evolves with interaction outcomes. Comparing with the traditional "resource" based view, it allows us to understand the dynamic process of trust and coordination.

- An explanation of the coexistence of low trust and 
high trust equilibrium-It shows that the high trust cannot be automatically achieved. It also brings implications on how to mediate the co-evolution of trust and coordination.

- An EGT model-It provides a rigorous way to assess the possibility of recurrent coordination failure in the long run, and a workbench for future work by incorporating various other socio-technical factors, such as network structure and individual differences $[20,21,22]$.

The rest of the paper proceeds as follows. Section 2 introduces the theoretical background. Section $3 \& 4$ specify the conceptual interaction process and details of the model. Section $5 \& 6$ present the analyses towards the model and the main propositions. Section $7 \& 8$ discuss the related issues and conclude the paper respectively.

\section{Background}

\subsection{Trust and Coordination}

Trust is a complex construct. There are numerous attempts to define and conceptualize it. Among many such efforts, one stream is to conceptualize it in terms of rational action choice in the interaction between trustee and trustor. Specifically, we adopt the definition in [23]: trust is "the willingness of a party to be vulnerable to the actions of another party based on the expectation that the other will perform a particular action important to the trustor, irrespective of the ability to monitor or control that other party.” (pp. 712).

Coordination is essential to regulate collaborations to achieve large system goal. It has been studied in multiple disciplines [4]. Coordination can be formal or informal, and influenced by various factors ranging from organization design [24] to techniques of managing task interdependencies. GSD projects are full of various task interdependencies, hence the coordination on work is crucial to team success $[25,5]$. Besides, the effectiveness of coordination may also influence the quality of software [26]. Studies such as [27] discuss the possibility of improving coordination via organization design.

Conventionally, trust is considered as a precondition for effective coordination [3]. For instance, [28] claimed that trust would reduce coordination conflicts; Yuan et al [29] argued that trust is one of the antecedents of coordination effectiveness in an open source project. But most of them do not consider the dynamics between trust and coordination. They investigate a static "snapshot" rather than a dynamic process of trust and coordination. Even in the literature that studies on-going trust, such as [12], trust is treated as "static". Furthermore, the majority of literature on the relationships between trust and coordination heavily relies on field observations. While they do well in collecting real-world data, the knowledge they produce is hard to be cross-examined, generalized and extended. Moreover, it is almost impossible to draw quantifiable predictions on long-term dynamics.

\subsection{Trust as Behavioral Constraints}

As a "belief", trust introduces behavioral constraints [16]. BPC approach assumes that an individual acts according to a specific set of feasible actions which depend on her preferences. Constraints resulting from belief impose further limitations to this action set. Hence, trust impacts individuals' behavior choices in interaction. Moreover, people who have similar trust often share more feasible behaviors in common. In this paper, we make the bold assumption that all team members prefer effective coordination only with differences in how much stronger the coordination preference is relative to each individual's other personal preferences (expressed as the "idiosyncratic payoff" in the model, see section 4). This bold assumption is reasonable in our context since, for work situations that we focus on, when coordination is achieved, the payoffs are higher than when coordination fails. We rule out the possibility of public goods game in which players may prefer social loafing for the payoff might be better when using anti-social behaviors. To sum up, the feasible actions in dyadic interactions are determined by the externalization of trust beliefs.

\subsection{Coordination Game}

Coordination game is an abstraction of coordination in real-world interactions. The basic, non-dynamic, 2-player, symmetric coordination game is shown in Fig. 1. The payoff for individuals is identical when they coordinate on action Red or Blue. It has two pure Nash equilibria: (Red, Red) and (Blue, Blue), which represent two social efficient states. (Red, Blue) or (Blue, Red) fails to achieve coordination, and the payoffs of them are strictly worse than successful coordination. The coordination game can also involve many players and many strategies.

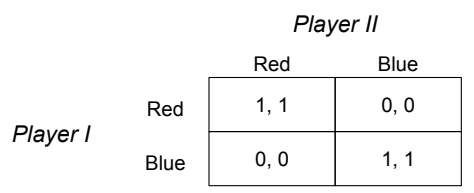

Figure 1. An example of coordination game.

When trust functions as constraints, it reduces the action space of forming potential coordination. Fig 2 . gives an example of a 2-player, 3-strategy coordination game. Ideally, there are 9 action pairs. However, each player can only select actions from 2 of the 3 strategies 
due to the trust restriction. Player I can choose action from $\left(x_{1}, x_{2}\right)$ while Player II can choose $\left(x_{2}, x_{3}\right)$. The number of action pairs is 4 (the "gray area" in the top-right of fig. 2). Trust alters the game structure and reduces the number of potential equilibria although $\left(x_{1}, x_{1}\right)$ and $\left(x_{2}, x_{2}\right)$ do provide better outcomes.

\begin{tabular}{|c|c|c|c|c|}
\hline & \multicolumn{3}{|c|}{ Player II } \\
\hline & & $x_{1}$ & $x_{2}$ & $x_{3}$ \\
\hline \multirow{3}{*}{ Player I } & $x$ & $1+0.8,1+0.8$ & $0.8,0$ & $0.8,0.8$ \\
\hline & $x_{2}$ & $0,0.8$ & $1+0,1+0$ & $0,0.8$ \\
\hline & $x_{3}$ & $0.8,0.8$ & $0.8,0$ & $1+0.8,1+0.8$ \\
\hline
\end{tabular}

Figure 2. Trust as behavioral constraints.

\subsection{Adaptive Play}

Young [17] proposed the Adaptive Play to describe the evolution of social institutions. It allows us to predict which solutions will be chosen in the long run by self-interested agents with limited information and resource, hence offers a good solution for modeling co-evolution of trust and coordination. It aims to describe " a world in which people base their decisions on limited data, use simple predictive models, and sometimes do unexplained or even foolish things"[18]. Basically, the adaptive play reflects a decision maker's "Bounded Rationality" [30] in real-world interaction.

In each round, each player uses simple dynamics to decide which strategy to play. It assumes that each player has limited memory $(m)$ about past rounds and a sample with size $s$ over $m$. The decisions are not necessarily to be optimal, which reflects the real decision-making situations with limited information processing ability. For the decision in current play relies on a limited number of prior dynamics, the whole process is a finite Markov process. In this process, a state in period $t$ can be represented by the $m$-history of $t$. The generic process is named as $P^{0}$. For this process, an absorbing set is a set of states that are all reachable from one another but cannot reach any states outside of the set. Due to the differences in initial states, there may be over one absorbing set. However, if adaptive play converges to an absorbing state, clearly the game must have a strict pure Nash equilibrium in pure strategies. This process may be perturbed by allowing agents to make mistakes.

\section{Conceptual Interaction Process}

Drawing from prior literature on empirical studies of trust and coordination in GSD teams (e.g. [31, 32, 2, 33, $34,35,36,37]$ ), we can summarize the following five simple yet prevalent insights:

1. Team members can be classified into subgroups according to their baseline trust. When collaboration begins, they tend to behave according to baseline trust. 2. The different trust levels restrict options of behaviors. 3. The mismatches between two subjects' behaviors lead to coordination failure in mutual interaction.

4. Although the baseline trust may not change too much, individuals update their situational trust according to the interaction outcomes.

5. Even for a team with long collaboration history, trust still varies.

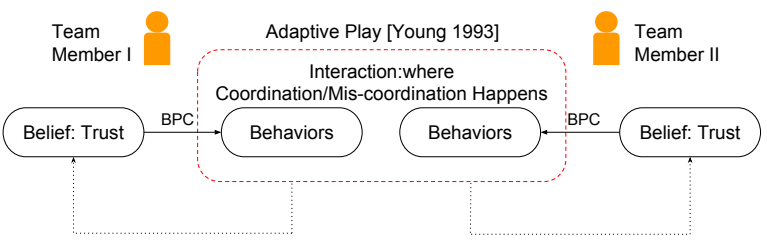

Figure 3. The conceptual dyadic interaction process.

We develop a conceptual interaction process (Fig. 3) focusing on coordination and trust. Let us assume all interactions in a GSD team are dyadic (other cases can be modeled as a set of dyadic interactions [19]). Before an interaction, a player only has limited, fragile information. It may be extracted from her own (or others') histories with those who are similar to her opponent. She then identifies the most probable behavior of her opponent by sampling the limited past histories. She would select a behavior from the action set shaped by her trust according to her assumption of her opponent's action. We do not care about the details about how trust beliefs restrict behaviors. Instead, we simply assume there is an invisible psychological belief externalization process to generate observable actions. Once both players have formed their actions, the interaction starts. The coordination can be either success or failure in this round. The players may review and adapt their trust beliefs in future according to coordination outcomes.

\section{The Model}

Our model $\mathcal{G}$ is a game consists of following elements: Player. There are $n$ players in $\mathcal{G}$. We assume that there is no new player entering or leaving. The set of players is denoted by $N$, and indexed by $i$. So, $n_{i}$ denotes a player.

Trust Levels. Assume there are finite $K$ different trust levels and they form a ladder structure. Every player belongs to one level in any given period, denoted by $g \in G=\cup_{1 \leq k \leq K} g_{k}$. Each level represents a unique degree of trust (shared by its members) towards others. A player in $g_{k}$ is referred as $g_{k}$-member. The set of $g_{k}$-member is $N_{k}$ where $\left|N_{k}\right|=n_{k}$. A level is empty if it has no member $\left(n_{k}=0\right)$. Everyone knows her own trust level and others', but has no prior knowledge about the 
action sets associated to other levels.

Strategies. We assume a set $X$ with cardinality $L$ is the set of all actions that can potentially be used in a two-player round. A player of $g_{k}$ must choose action from the pure strategy set $X_{k} \in X=\cup_{1 \leq k \leq K} X_{k}$. So, we have:

Condition 1: Trust Constraints $A$ member of the trust level $g_{k}$ in period $t$ can only make behavioral choice from the action set: $X_{k}$, when interacting in current period.

Such restriction might come about through the externalization of trust perception (fig. 2). If $\left|X_{k}\right|=L$, $g_{k}$-members have no restriction to their actions resulting from trust. However, this strict high trust level does not necessarily exist. In fact, it is almost impossible to find such a trust level in any team.

\subsection{Individuals' Payoff}

The coordination impact both parties' payoffs. (1) presents the payoff function which satisfies the von Neumann-Morgenstern utility theorem [38]:

$$
u\left(x_{i}, x_{-i}\right)= \begin{cases}P \_ \text {Interaction }+\pi_{i, x}, & \text { if succeeds } \\ \pi_{i, x}, & \text { else }\end{cases}
$$

$P$ Interaction represents the payoff from successful coordination. We assume it is 1 if the coordination succeeds, and the players only receive idiosyncratic payoff in all other cases. $\pi_{-} i, x$ is the idiosyncratic payoff of each player, which reflects the preference on different actions of each trust level. Assuming all players prefer successful coordination, $\pi_{i, x}<1$.This ensures that the whole payoff is determined by the payoff from interaction, and excludes the anti-social behaviors.

\subsection{Adaptive Play Process}

Round. The game is played in discrete periods, denoted by $t=1,2,3, \ldots T$. In each round, two players are randomly selected to participate in a social interaction. Learning Dynamics. Adaptive play assumes a player has limited information and information processing ability. She plays game by examining a limited sample of history, and select action according to her expectation to her opponent's action in this period. She forms expectation based on the prior plays of members of her partners' trust group. Suppose two players were from trust levels $g_{k}$ and $g_{j}$, we name this interaction as $j$ - $k$ interaction. Before the play starts, they can access limited number $m$ of previous $j-k$ interactions (namely, $j-k$ history) due to the limitation of their memory. $j$ - $k$ history defines the current state at $t$ , and the play in this round carries the process to another state $^{2}$. Table 1 shows the formal denotations and their meaning. To form the expectation, the player samples $s$

\footnotetext{
${ }^{2}$ The play in this round generates the newest $j$ - $k$ interaction instance which replaces the oldest one in the memory.
}

Table 1. Denotations and Meanings (memory).

\begin{tabular}{cl}
\hline \multicolumn{1}{c}{ Meanings } \\
\hline$x_{j-k}^{t,-1}$ & $\begin{array}{l}\text { The most recent action of } g_{k} \text {-member in } j \text { - } k \\
\text { interaction }\end{array}$ \\
\hline$x_{j-k}^{t,-m}$ & $\begin{array}{l}\text { The m-th most recent action of } g_{k} \text {-member in } \\
j \text {-k interaction }\end{array}$ \\
\hline $\mathbf{x}_{j-k}^{t,-1}$ & $\begin{array}{l}\text { The most recent action pair of both } g_{j} \text {-member } \\
\text { and } g_{k} \text {-member in } j \text { - } k \text { interaction }\end{array}$ \\
\hline $\mathbf{x}_{j-k}^{t,-m}$ & $\begin{array}{l}\text { The m-th most recent action pair of both } \\
g_{j-m e m b e r} \text { and } g_{k} \text {-member in } j \text { - } k \text { interaction }\end{array}$ \\
\hline$h_{j-k}^{t}$ & $\begin{array}{l}\left(\mathbf{x}_{j-k}^{t,-m}, \ldots . \mathbf{x}_{j-k}^{t,-1}\right): \text { The collection of action pairs } \\
\text { in the } m \text { previous } j-k \text { interactions }\end{array}$ \\
\hline
\end{tabular}

from $j$ - $k$ history $\left(h_{j-k}^{t}\right)$ in round $t$. The other player in this interaction does the similar thing.

After the sampling, the players locally maximize their expected payoff in the current period. If no mistake, both two players choose the best replies according to their resulting sample proportion. In section 5.3, making mistake becomes possible. We assume the probability of making a mistake is $\epsilon$. Fig. 4 describes the process.

\subsection{Convention vs. Coordination Failure}

Convention is the best strategies emerging from interactions [39]. It is a pure Nash equilibrium that has been played by the entire population for the periods in which memory keeps [17]. Obviously, $j$ - $k$ interaction is a Markov process defined on $\prod^{m}\left(X_{j}, X_{k}\right)$. The state in $t+1$ $\left(h_{j-k}^{t+1}\right)$ is determined by the state in $t$. If the convention has been formed on action $y$, the history at $t$ should be: $h_{j-k}^{*}=\left(\mathbf{x}_{j-k}^{t,-m}, \ldots \mathbf{x}_{j-k}^{t,-1}\right)=((y, y) \ldots(y, y))$. While players in all $j$ - $k$ interaction keep playing this convention, the interaction payoff equation will be guaranteed. For the idiosyncratic payoff is strictly less than 1 , the convention always brings optimal payoff. Recurrent Coordination Failure is another extreme case. In a state of this class, players cannot learn any useful knowledge from the history to form a strategy to get interaction payoff. Hence, we define it as: $h_{j-k}^{*}=\left(\left(x_{j-k}^{t-m}, x_{k-j}^{t-m}\right), \ldots\left(x_{j-k}^{t-i}\right.\right.$, $\left.\left.x_{k-j}^{t-i}\right), \ldots\left(x_{j-k}^{t-1}, x_{k-j}^{t-1}\right)\right)$ if $x_{j-k}^{t-i} \notin X_{k}$ and $x_{k-j}^{t-i} \notin X_{j}$. No $j-k$ interaction will achieve coordination in future.

\section{Coordination in the Long Run}

We will explore if members of different trust levels could eventually develop effective coordination (conventions) with each other. By answering this question, we reveal the trend of potential recurrent coordination failure in the long run. In this section, we assume that nobody changes her initial trust level. The analysis follows the approach in [18], which allows both undesirable recurrent coordination failure) and desireable 


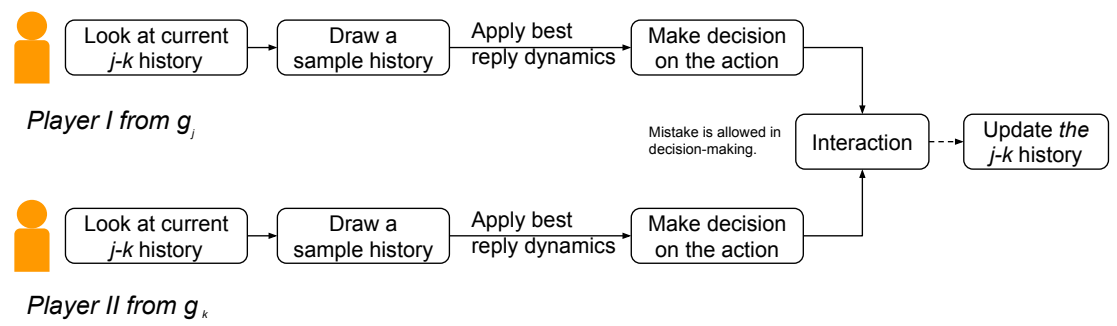

Figure 4. The process of play in period $t$.

conventions as stochastic stable states. Essentially, we find that:

$<$ Coordination in the Long Run $>$ If $j=k$, a convention for $j$ - $k$ interactions will be achieved. If $j \neq k$, the possibility of recurrent coordination failure does not only exists but also may be very high for j-k interactions in the long-run regardless of the configurations of $j-k$.

According to Fig. 4, players in a $j$ - $k$ interaction make their decisions by sampling from $j-k$ history only. Hence, what will happen in a $j-k$ interaction is independent of other levels if no player changes her trust level. To simplify the discussion and without the loss of generality, we focus on a special instance of $j$ - $k$ interaction, which is the 1-2 interaction. This simplification does not influence the generality of the conclusions, for 1-2 only indicates this pair of players from two different trust levels.

\subsection{Coordination in the Unperturbed Process}

We use $P_{1-2}^{0}$ to denote the unperturbed process (no mistake). In $P_{1-2}^{0}$, players form both trust level strictly follow the best reply dynamics. In period $t$, current state $w$ is determined by the history in $t$, i.e. $w^{t}=h_{1-2}^{t}$, and the state space is defined by the m-fold product of $X_{1}$ and $X_{2}$. According its definition, conventions should be the actions that are acceptable for both players. Hence, all potential conventions form a set $\mathcal{C}=X_{1} \cap X_{2}$ indexed by $c$. If $\mathcal{C}=\phi$, no convention could be reached no matter how long this process continues, the only outcome is recurrent coordination failure. Hence, we concern more about if the process reach conventions when $\mathcal{C} \neq \phi$.

The payoff structure in (1) contains two components. A player will always get an idiosyncratic payoff. However, she may miss the interaction payoffs. It is possible that the maximal idiosyncratic payoff is so attractive for a player with bounded rationality that she keeps playing it and eventually is trapped in recurrent coordination failure. Let $\widetilde{X_{k}}=\left\{\widetilde{x} \mid \widetilde{x_{i, k}} \in \operatorname{argmax} \pi_{i, x}\right\}$ refers the set of actions maximize a players idiosyncratic payoff. Applying this heuristic, we define a new relationship named "Small-Different".

Two trust levels $\left(g_{j}\right.$ and $\left.g_{k}\right)$ are Small-Different iff $\widetilde{X_{j}} \cap$ $\widetilde{X_{k}}=\phi$. and $X_{1} \bigcap X_{2} \neq \phi$. If $X_{1} \bigcap X_{2}=\phi$, they are

\section{Total-Different.}

Basically, Small-Different indicates two trust levels share no preferred action but do have some mutual acceptable actions to be potential conventions. In fig. 2, suppose Player $I$ in $g_{1}$ and Player $I I$ in $g_{2}$, possible actions for $g_{1}$ is $\left\{x_{1}, x_{2}\right\}$ and $x_{1}$ is preferred while possible actions for $g_{2}$ is $\left\{x_{2}, x_{3}\right\}$ and $x_{3}$ is preferred. According to above definition, $g_{1}$ and $g_{2}$ are Small-Different but not Total-Different, and $x_{2}$ is potentially to be a convention.

If 1-2 are Small-Different trust levels, the recurrent coordination failure can be defined as a set of states of $\left(\widetilde{X_{1}} \times \widetilde{X}_{2}\right)^{m}$, which indicates no any mutual acceptable actions appear in the history. For above example, if a history was filled with $\left(x_{1}, x_{3}\right)$, it would be recurrent coordination failure. For a Markov process, it is an absorbing set of states that each of them can be accessed by peer states in this set (state transition probability is positive in a finite number of periods) but no one can access the states outside this set (no positive state transition probability). We propose the following proposition:

Coordination Proposition. Suppose $\beta=s / m$, if $\beta \leq$ $1 / 2$, process $P_{j-k}^{0}$ would almost always reach: 1 . A convention if $j-k$ are not Different; 2 . Either $a$ convention or a recurrent coordination failure if $j-k$ are Small-Different; 3. A recurrent coordination failure if $j-k$ are Total-Different.

Note that beta $\leq 1 / 2$ is not a strict bound, it is used to guarantee the randomness of the Markov process. A detailed proof is in the online supplement (https: $/ / g \circ o . g 1 / \operatorname{RywBr} 2)$. If $j=k$, this proposition is still valid, because $j=k$ is actually a special case of the first case where $\widetilde{X_{j}} \cap \widetilde{X_{k}}=\widetilde{X_{j}}=\widetilde{X_{k}}$. This property shows that the long-term effectiveness of coordination between players from the same trust levels will definitely reach a convention (successful coordination).

\subsection{What Happens to the Concrete Example?}

Let us go back to the example in fig. 2. In any single round, if both players choose action $x_{2}$, the coordination will be reached. So, the question is: can they learn this social-efficient equilibrium and keep playing it in 


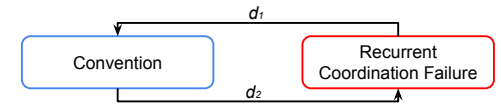

Figure 5. Convention $\leftrightarrow$ recurrent coordination failure.

the long run? Coordination Proposition cannot give a deterministic conclusion since Player $I$ and II are from Small-Different trust levels. We only know that the interactions between them reaches either convention or recurrent coordination failure. The next sub-section answers this question by studying the perturbed process in which players' small mistakes are allowed.

\subsection{Coordination in the Perturbed Process}

The interaction of players from Small-Different levels may result in recurrent coordination failure. But if the possibility is low, we can draw stronger and more favorable conclusions. However, following analysis will show that this is not true. We resort to the stochastic stability framework of the perturbed process to uncover whether recurrent coordination failure is frequent. We refer to the perturbed process as $P_{1-2}^{\epsilon}$, where $\epsilon$ is the probability of making a mistake when forming action. When a player makes a mistake, she randomly selects an action. Assuming $\epsilon$ approaches 0 , we can draw conclusions on the asymptotic stability of the process.

Mistakes drive the switch between convention and recurrent coordination failure. If the "basin of attraction" of the latter is large enough, it is very hard to switch to conventions. Intuitively, idiosyncratic payoffs impact the "basin of attraction." Fig. 5 describes the transitions between convention and recurrent coordination failure. We define $d_{1}$ as the minimal resistance from recurrent coordination failure to convention ${ }^{3}$, and $d_{2}$ is the maximal resistance from convention to recurrent coordination failure. If recurrent coordination failure becomes stable, $d_{1}$ must be greater than $d_{2}$.

Calculate $d_{1}$ and $d_{2}$. Consider a transition from recurrent coordination failure to convention. In period $t, P_{1-2}^{\epsilon}$ is in recurrent coordination failure, hence, any sample $x^{\prime}$ from $h_{1-2}^{t}$ satisfies $x^{\prime} \in X_{1} \cap X_{2}=\phi$. Now, suppose in next $q$ periods, $g_{2}-$ member makes mistake by selecting $x_{c} \in X_{1} \cap X_{2}$ continuously. In period $t+q+1$, the probability for a sample contains all $q$ play of $X_{c}$ is positive. Here, the expected playoff is $\frac{q}{s}+\pi_{i, x_{c}}$. To ensure this player plays $x_{c}$, the expected payoff of playing $x_{c}$ should be greater than playing $x$, we need:

$$
\frac{q}{s}+\pi_{i, x_{c}} \geq \pi_{i, x^{\prime}}
$$

Hence, we have $q=\left\lceil s\left(\max _{x \in X_{1}} \bigcap \overline{X_{2}} \pi_{i, x}-\pi_{i, x_{c}}\right)\right\rceil$.

\footnotetext{
${ }^{3}$ Non-strictly, "resistance" can be viewed as the number of mistakes required for a specific transition.
}

Simply extend it to all possible actions, we have:

$$
d_{1}=\left\lceil s\left(\min _{i \in N_{1}}\left(\max _{x \in X_{1} \bigcap \overline{X_{2}}} \pi_{i, x}-\max _{x_{c} \in X_{1} \bigcap X_{2}} \pi_{i, x_{c}}\right)\right)\right\rceil
$$

By similar reasoning, we can calculate $d_{2}$.

$$
d_{2}=\left\lceil s\left(1-\max _{i \in N_{1}}\left(\max _{x \in X_{1} \bigcap \overline{X_{2}}} \pi_{i, x}-\max _{x_{c} \in X_{1} \bigcap X_{2}} \pi_{i, x_{c}}\right)\right)\right\rceil
$$

Now, we define two new measures for idiosyncratic payoff to formally established this relationship.

$$
\begin{aligned}
& \pi^{+}=\max _{i \in N_{1}}\left(\max _{x \in X_{1} \bigcap \overline{X_{2}}} \pi_{i, x}-\max _{x_{c} \in X_{1} \bigcap X_{2}} \pi_{i, x_{c}}\right) \\
& \pi^{-}=\min _{i \in N_{1}}\left(\max _{x \in X_{1} \bigcap \overline{X_{2}}} \pi_{i, x}-\max _{x_{c} \in X_{1} \bigcap X_{2}} \pi_{i, x_{c}}\right)
\end{aligned}
$$

If $d_{1}>d_{2}$, according (3) and (4), we now have

$$
d_{1}>d_{2} \Rightarrow\left\lceil s \pi^{-}\right\rceil>\left\lceil s\left(1-\pi^{+}\right)\right\rceil
$$

$s$ is reducible in above inequation, hence we have:

$$
\pi^{-}+\pi^{+}>1
$$

Given $\pi^{+}$and $\pi^{-}$, we propose following proposition:

$\epsilon$-Coordination Proposition. Suppose $\beta=s / m$, if $\beta \leq$ $\overline{1 / 2}$, and $j-k$ are Small-Different trust levels. If $\pi^{+}$ $+\pi^{-}>$P_Interaction, process $P_{1-2}^{\epsilon}$ would have recurrent coordination failure as its unique stochastically stable states.

The discussion in this section sketches the proof of the proposition. What does this proposition imply? It is similar to "black hole" theory in physics, the higher $\pi^{+}$is, the interaction is more likely to enter the recurrent coordination failure, and the higher $\pi^{-}$is, the more difficult to leave. When they are big enough, nothing could leave these states. They form the "event horizon" of the recurrent coordination failure. $\pi^{+}+\pi^{-}>$ P_Interaction is very weak, the recurrent coordination failure may be common. Let us answer the question about the concrete example in Fig. 3. For the preferences are "symmetric" (the idiosyncratic payoffs $\pi_{m}$ of preferable action are same for both players), $\pi^{+}=\pi^{-}=0.8$. Hence, $\pi^{+}+\pi^{-}=1.6>1$. So, $P_{1-2}^{\epsilon}$ will be stuck in the recurrent coordination failure states.

\section{Dynamic Trust Evolution}

When people update their trust, what will happen? Since cross-level interactions often raise the recurrent coordination failure, this question can be expressed in another form: is it possible that all levels to be merged into one high trust to avoid coordination failure? We now 
show a team that has $K(K \geq 3)$ trust levels would almost always evolve into a team has two levels. Moreover, one of them will be a low trust level, while the other is the higher trust level dominating the lower. This evolution may take a long period, or even longer than a team's life cycle. Basically, we demonstrate that it is unlikely for a large enough team to achieve a unified high trust level only motivated by coordination benefits.

Suppose there are three trust levels, one is strictly higher than the other two. We will show the high trust level and one of the other two would stably coexist in the long run. Once this is established, we can reach similar argument for $N$-level team, which would eventually merge into three-level or two-level setting if the convergence occurs.

\subsection{Individual Trust Update}

Update Timing. At the beginning of each round, a player has an opportunity to review her trust and decide whether or not to update her belief and corresponding action profile with a non-negative probability $\varphi$ (change rate). We assume the "change" is costless for it is a mental activity. This change is determined by the evaluation of potential payoffs based on best-reply dynamics (i.e., whether the change yields better immediate payoffs).

Process. For players are allowed to change their trust, the distribution of trust levels is dynamically relevant to their decisions. The state in current period $t$ should contain the history of play in this period and the distribution of players over the trust for the change of trust level between $t-1$ and $t$. We name the current state as $w$, which is in a much larger finite state space $W$. Now, the process is assumed to start from an initial state $w^{0}$. When the process starts, there are already $m$ plays in each $j-k$ interaction as the prior knowledge. $P_{w, w^{\prime}}$ denotes the transition probability between $w$ and $w^{\prime}$. Hence, we define the adaptive process as a finite Markov process, whose transition probability matrix is relevant to the parameter $\varphi$.

\subsection{The Convergence of Trust Levels}

So, what will happen for a team with three trust levels in the long run if people could change their trust levels? To simplify the discussion, we first make an assumption that every interaction in that setting is a $2 \times 2$ game, and the actions are limited to $X=\left\{x_{1}, x_{2}\right\}$. Then, we can simply define three trust levels $\left\{g_{1}, g_{2}, g_{3}\right\}$ indirectly, which $g_{1}$ only allows action $x_{1}, g_{2}$ only allows action $x_{2}$, and $g_{3}$ allows both of them. Besides, we assume that each player prefers one action $\left(x_{1}\right.$ or $\left.x_{2}\right)$, and both two actions have at least one player who prefers it.

We define a set of states that all players prefer $x_{1}$ most are from $g_{1}$ while the others who prefer action $x_{2}$ already converge into $g_{3}$. Obviously, in these states, the group $g_{2}$ does not exist any longer. These states can be described as $\widetilde{W}_{1}=\left\{w \mid N_{2}=\phi, N_{1}=\widetilde{N}_{1}\right.$, and $\left.N_{3}=\widetilde{N}_{2}\right\}$. Similarly, we can define another set of state that all players prefer $x_{2}$ are in $g_{2}$ while the others who prefer action $x_{1}$ most form $g_{3}$. In this case, the group $g_{2}$ is eliminated. Formally, these states are $\widetilde{W}_{2}=\left\{w \mid N_{1}=\phi, N_{2}=\widetilde{N}_{2}\right.$, and $\left.N_{3}=\widetilde{N}_{1}\right\}$. Assume $\widetilde{W}=\widetilde{W}_{1} \cup \widetilde{W}_{2}$.

Now, let us suppose the interaction starts with $t=0$. We use $\mu^{t}\left(w \mid w^{0}\right)$ to denote the relative frequency that a state is reached by the unperturbed process $P^{0}$ in a finite number of periods. With the periods increase, it is reasonable to assume that $\mu^{t}\left(w \mid w^{0}\right)$ almost always approaches to the probability distribution $\mu^{t \rightarrow \infty}\left(w \mid w^{0}\right)$. In this process, if the $\mu^{t \rightarrow \infty}\left(w \mid w^{0}\right)$ has positive probability in some specific states, these states would be legal absorbing states of a given process. The following proposition establishes the convergence.

Convergence Proposition. For a team that has three trust levels, if the dynamic process begins in any state $w^{0}$ and all three trust levels are not empty in this state. Suppose the sample size is no more than half of the memory size, t approaches $\infty$, and $w \in \widetilde{W}$ we have:

$$
\mu^{t \rightarrow \infty}\left(w \mid w^{0}\right) \rightarrow 1 \text {, while } \varphi \rightarrow 0
$$

The proof of the convergence proposition is in the same online supplement (https://goo.gl/ RywBr2). The proposition indicates that when individuals seldom change their trust levels, the high level of trust (here, $g_{3}$ ) will be almost always (the probability approaches 1) in a companion of exactly one less trust level, although we cannot determine which less trust level will survive. However, we can determine which group survives by observing the convention of the interaction whose both parties are from $g_{3}$. If the convention is on $x_{1}$, the $g_{2}$ has been eliminated, and vice versa. In both situations, high-trust individuals yield to low trust conventions to avoid coordination failure. Even though, this final two-level trust cannot be guaranteed to be reached in the real life due to many practical limitations. We will show an example in next section. Please note that our focus is NOT to demonstrate all teams will achieve a two-level trust state in a reasonable time frame, but to demonstrate:

"the High DIFFICULTY (if not impossibility) of achieving a single-level trust state in acceptable period".

\subsection{Trust Convergence in GSD Teams}

Considering a scenario described in the left part of fig. 6, there is a team located in two different places. In each location, there are three different trust levels. Due to the lack of memory of cross-location interaction between 


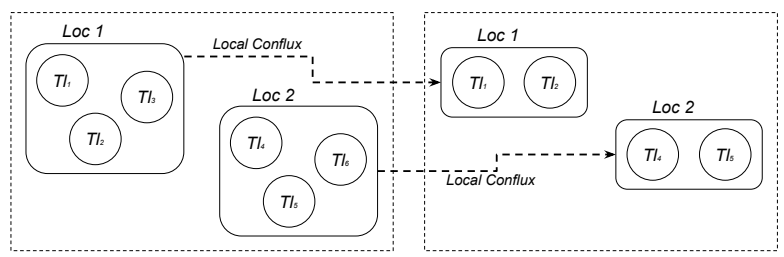

Figure 6. Local-Preferred Trust Convergence.

the members these two locations, the process of global convergence may be very slow. In some situations, the global level convergence may fail to be achieved. We will not formally present a new model to demonstrate this. Instead, we discuss this point in narratives.

Suppose the two locations are Loc1 and Loc2, and in a specific period $t>0$, the whole system is in a special state that all possible interactions between Loc1 and Loc2 are in recurrent coordination failure, and the all possible interactions within Loc1 or Loc2 are in some conventions. Starting from this state, it is more likely that there is an independent convergence process in each location that makes the global state turn out to be the right half of fig. 6 . In this state, the probability of convergence at the global level is small for there are few opportunities for both locations to build diverse enough memory, which leads to interactions across locations are randomly determined. Individuals are more likely to make decisions according to the idiosyncratic payoffs, which leads to recurrent coordination failure $(\epsilon$-Coordination Proposition).

\section{Discussion and Implications}

\subsection{How Trust Influences Coordination?}

Different trust levels matter. In the case of no trust level switch, the conventions almost always can be achieved if people in interaction are not from Small/Total-Different levels. For the players are from Small-Different levels, their interactions result in recurrent coordination failure in a high probability. So, we can develop a conceptual process of the co-evolution of trust and coordination with the behavior constraints. The improved trust reduces the behavior constraints and makes more behaviors mutually acceptable, thereby contributes to forming conventions on these behaviors. Once the conventions have been built, what will happen? Ceteris paribus, the system would be in stable states where people simply repeat the conventions to achieve coordination. Nobody has the incentive to change her behaviors since she already achieves the optimal payoff and is able to keep enjoying it. We argue that it is almost impossible for the whole team to share high trust for there is no intensive for people in lower-trust to improve their trust. The improved trust will not lead to better coordination for high trust people learned to conform to them to avoid coordination failure in the 2-level setting.

Even when nobody trusts any others, there are still chances for good coordination. Under this circumstance, the behavior choices are very limited. The effective coordination is achieved through strict control over behaviors. E.g., in a GSD team where team members have no trust towards each other, people have to spend extra time to do unnecessary work to fulfill others' expectations. Comparing with the conventions formed in high trust setting, this is less favorable because the effectiveness of coordination is achieved at the price of extra intra-organization transaction cost [40]. This partially explains why high-trust teams perform better.

\subsection{Team vs. Individual}

The $\epsilon$-Coordination proposition indicates that long-term recurrent coordination failures are closely related to the "basin of attraction" created by idiosyncratic payoffs. According to equation (6), large idiosyncratic payoffs are more likely leading to recurrent coordination failure for both $\pi^{+}$and $\pi^{-}$would increase if idiosyncratic payoffs become larger. If we change the idiosyncratic payoff in fig. 2 from 0.8 to 0.2 , no recurrent coordination failure will survive. Hence, coordination achieves by lowering idiosyncratic payoffs which represent one's own preferences at a specific trust level. When a team has high team cohesion, its members' idiosyncratic payoff would be less important for them, sometimes, they may even sacrifice their own preferences for their team [41]. Therefore, if the team cohesion is high, recurrent coordination failures will be less likely even when the trust may vary a lot. You may not trust another team member, but if you concern team's achievements more than your own preferences, you may be willing to adapt to her by (partially) giving up some idiosyncratic payoff. Thus, while the trust varies, increasing the team cohesion might be a remedy for coordination failure.

\subsection{Managerial and Design Implications}

Conventions are social controls [42]. Social control is often viewed to be contradictory to building trust. But, our study shows the trust itself is also a type of social control, e.g., the high-trust people often need to conform to the low-trust group's convention in cross-group interactions. The duality of trust and social control has been discussed in literature [43], particularly in virtual organization context, as [44] highlighted:

Trust and control have typically been viewed as opposites or substitutes, ... We argue that trust and control are closely intertwined and often mutually reinforcing approaches to managing distributed work... 
Our results also partially support a more aggressive view than balancing trust and control [45]. That is: trust may be a type of social control. The benefit of this view is that it enables us to investigate trust and social control in a unified framework. Moreover, the coordination, as the results of social control, can be better predicted by the presence of trust. How to leverage social control mechanisms to enhance trust is also an interesting issue.

Researchers have developed many computational tools to improve trust in GSD teams. However, they may lead to trust decreases. For example, if faithfully displaying an individual's past collaborative traces, it is likely that the "bad history" would also be shown. Therefore, trust may be hurt rather than improved. But, the "hurt" may help build reasonable behavior expectations. The expectations help to develop conventions or social controls, and hence reduce coordination failures [39]. If a tool only displays one's positive history but hides the negatives, the manipulated trust may lead to coordination failure by reinforcing wrong expectation [46]. Unless the trust is unanimously high among all members which is unlikely [47], the risk of concurrent coordination failure may always exist.

Let us rethink the role of computational tools in supporting trust building. Promoting trust is good but not necessarily always brings better coordination. Trust improvement may make people become overconfident. The interactions among tools, trust, social learning, and coordination are very complex. Based on our results and Arrow's arguments on trust in large teams [46], we hypothesize that trust tools promote trust in a small team or where the systematic risks are low, hence improve coordination. When a team is large, effective coordination more relies on forming expectations and social learning rather than trust. In the latter case, tools are valuable to enhance understandings and to facilitate learning between a trustee and a trustor, which helps develop the conventions during interactions. Since facilitating the learning of remote collaborators' behaviors is crucial for improving the coordination, Collaboration tools should enable the information cascading over different locations.

\section{Conclusions and Future Work}

In this paper, we develop an Evolutionary Game Theory (EGT) model to describe the co-evolution of trust and coordination in Global Software Development (GSD) teams. The analyses of the model reveal how trust and coordination co-evolve in a GSD team. Team members with different levels of trust can form conventions to avoid coordination failure, however, even interactions of individuals from Small-Different trust levels may end with the recurrent coordination failure. Moreover, the high-trust people tend to conform to the low-trust people's convention while the latter has few incentives to improve their trust in the long run. Hence, it is difficult to eliminate low trust in a large enough GSD team. The model builds a theoretical foundation for investigating the co-evolution of trust and coordination and can be easily extended to incorporate various individual and organizational factors.

We plan to develop simulations to identify the details of the co-evolution process. Besides, we will incorporate network features into the current model. We will also extend the model to incorporate other social factors such as personality, organizational role, and so on. All mathematical models, no matter how sophisticated they are, are imperfect abstractions of the real-world practices. The external validity should be examined through empirical evidence. We have planned empirical field experiments to validate the model and related propositions directly.

\section{References}

[1] B. Al-Ani, M. J. Bietz, Y. Wang, E. Trainer, B. Koehne, S. Marczak, D. Redmiles, and R. Prikladnicki, "Globally distributed system developers: their trust expectations and processes," in Proc. CSCW'13, pp. 563-574, ACM, 2013.

[2] P. Hinds, Distributed work. Cambridge, MA: MIT Press, 2002.

[3] G. M. Olson and J. S. Olson, "Distance matters," Hum.-comp. Inter, vol. 15, no. 2, pp. 139-178, 2000.

[4] T. W. Malone and K. Crowston, "The interdisciplinary study of coordination," ACM Computing Survey, vol. 26, pp. 87-119, Mar. 1994.

[5] J. Herbsleb, "Building a socio-technical theory of coordination: Why and how (outstanding research award)," in Proc. FSE'16, pp. 2-10, ACM, 2016.

[6] E. Salas, D. Sims, and C. Burke, "Is there a "big five" in teamwork?," Small group research, vol. 36, no. 5, pp. 555-599, 2005.

[7] S. L. Jarvenpaa and D. E. Leidner, "Communication and trust in global virtual teams," Journal of Computer-Mediated Communication, vol. 3, no. 4, pp. 0-0, 1998.

[8] R. Zolin and P. J. Hinds, "Trust in context: The development of interpersonal trust in geographically distributed work," Trust and distrust in organizations: Dilemmas and approaches, pp. 214-238, 2004.

[9] R. Zolin, P. J. Hinds, R. Fruchter, and R. E. Levitt, "Interpersonal trust in cross-functional, geographically distributed work: A longitudinal study," Information and organization, vol. 14, no. 1, pp. 1-26, 2004.

[10] M. A. Marks, J. E. Mathieu, and S. J. Zaccaro, "A temporally based framework and taxonomy of team processes," Academy of management review, vol. 26, no. 3, pp. 356-376, 2001.

[11] K. Schmidt, "Cooperative work and coordinative practices," in Cooperative Work and Coordinative Practices, pp. 3-27, Springer, 2008. 
[12] B. A. De Jong and T. Elfring, "How does trust affect on-going team? the mediation role of reflexivity, monitoring, and effort," Academy of Management Journal, vol. 53, no. 3, pp. 535-549, 2010.

[13] S. L. Jarvenpaa, T. R. Shaw, and D. S. Staples, "Toward contextualized theories of trust: The role of trust in global virtual teams," Information systems research, vol. 15, no. 3, pp. 250-267, 2004.

[14] D. M. Rousseau, S. B. Sitkin, R. S. Burt, and C. Camerer, "Not so different after all: A cross-discipline view of trust," Academy of management review, vol. 23, no. 3, pp. 393-404, 1998.

[15] P. Kanawattanachai and Y. Yoo, "Dynamic nature of trust in virtual teams," The Journal of Strategic Information Systems, vol. 11, no. 3-4, pp. 187-213, 2003.

[16] S. Bowles and H. Gintis, A Cooperative Species: Human Reciprocity and Its Evolution. NJ: Princeton Press, 2011.

[17] H. P. Young, "The evolution of conventions," Econometrica, vol. 61, no. 1, pp. 57-84, 1993.

[18] H. P. Young, Individual Strategy and Social Structure: An Evolutionary Theory of Institution. NJ: Princeton Press, 1998.

[19] Y. Wang and D. Redmiles, "Cheap talk, cooperation, and trust in global software engineering," Empirical Software Engineering, vol. 21, no. 6, pp. 2233-2267, 2016.

[20] S. P. Borgatti and P. C. Foster, "The network paradigm in organizational research: A review and typology," Journal of management, vol. 29, no. 6, pp. 991-1013, 2003.

[21] R. Reagans, E. Zuckerman, and B. McEvily, "How to make the team: Social networks vs. demography as criteria for designing effective teams," Administrative Science Quarterly, vol. 49, no. 1, pp. 101-133, 2004.

[22] G. Soda, A. Usai, and A. Zaheer, "Network memory: The influence of past and current networks on performance," Academy of Management Journal, vol. 47, no. 6, pp. 893-906, 2004.

[23] R. C. Mayer, J. H. Davis, and F. D. Schoorman, "An integrative model of organizational trust," The Academy of Management Review, vol. 20, no. 3, pp. 709-734, 1995.

[24] S. Faraj and Y. Xiao, "Coordination in fast-response organizations," Management Science, vol. 52, no. 8, pp. pp. 1155-1169, 2006.

[25] T. Gross, "Supporting effortless coordination: 25 years of awareness research," Computer Supported Cooperative Work (CSCW), vol. 22, no. 4-6, pp. 425-474, 2013.

[26] M. Cataldo, A. Mockus, J. A. Roberts, and J. D. Herbsleb, "Software dependencies, work dependencies, and their impact on failures," IEEE Transactions on Software Engineering, vol. 35, no. 6, pp. 864-878, 2009.

[27] K. Crowston, "A coordination theory approach to organizational process design," Organization Science, vol. 8, no. 2, pp. $157-175,1997$.

[28] R. S. Peterson and K. J. Behfar, "The dynamic relationship between performance feedback, trust, and conflict in groups: A longitudinal study," Organizational Behavior and Human Decision Processes, vol. 92, no. 1-2, pp. 102-112, 2003.

[29] M. Yuan, X. Zhang, Z. Chen, D. Vogel, and $\mathrm{X}$. Chu, "Antecedents of coordination effectiveness of software developer dyads from interacting teams: An empirical investigation," Engineering Management, IEEE Transactions on, vol. 56, pp. $494-507$, aug. 2009.
[30] H. A. Simon, Models of bounded rationality: Empirically grounded economic reason, vol. 3. MA: MIT press, 1982.

[31] J. Delhey, K. Newton, and C. Welzel, "How general is trust in most people? solving the radius of trust problem," American Sociological Review, vol. 76, no. 5, pp. 786-807, 2011.

[32] J. W. Driscoll, "Trust and participation in organizational decision making as predictors of satisfaction," Academy of management journal, vol. 21, no. 1, pp. 44-56, 1978.

[33] S. L. Jarvenpaa, K. Knoll, and D. E. Leidner, "Is anybody out there? antecedents of trust in global virtual teams," Journal of management information systems, vol. 14, no. 4, pp. 29-64, 1998.

[34] L. S. Wrightsman, Interpersonal trust and attitudes toward human nature, pp. 373-412. Measures of personality and social psychological attitudes, San Diego, CA, US: Academic Press, 1991.

[35] Y. Wang and D. Redmiles, "The diffusion of trust and cooperation in teams with individuals' variations on baseline trust," in Proc. CSCW'16, pp. 303-318, ACM, 2016.

[36] T. Yamagishi and M. Yamagishi, "Trust and commitment in the united states and japan," Motivation and emotion, vol. 18, no. 2, pp. 129-166, 1994.

[37] J. Zheng, E. Veinott, N. Bos, J. S. Olson, and G. M. Olson, "Trust without touch: jumpstarting long-distance trust with initial social activities," in Proc. CHI'O2, pp. 141-146, ACM, 2002.

[38] J. Von Neumann and O. Morgenstern, Theory of games and economic behavior (commemorative edition). Princeton university press, 2007.

[39] G. Mark, "Conventions and commitments in distributed cscw groups," Computer Supported Cooperative Work (CSCW), vol. 11, no. 3-4, pp. 349-387, 2002.

[40] O. E. Williamson, "The theory of the firm as governance structure: From choice to contract," The Journal of Economic Perspectives, vol. 16, no. 3, pp. 171-195, 2002.

[41] G. Van Der Vegt, E. Van De Vliert, and A. Oosterhof, "Informational dissimilarity and organizational citizenship behavior: The role of intrateam interdependence and team identification," The Academy of Management Journal, vol. 46, no. 6, pp. 715-727, 2003.

[42] K. Arrow, "Control in large organizations," Management Science, vol. 10, no. 3, pp. 397-408, 1964.

[43] G. Möllering, "The trust/control duality: An integrative perspective on positive expectations of others," International sociology, vol. 20, no. 3, pp. 283-305, 2005.

[44] M. O’Leary, W. Orlikowski, and J. Yates, “Distributed work over the centuries: Trust and control in the hudson's bay company, 1670-1826," in Distributed Work (P. J. Hinds and S. Kiesler, eds.), ch. 2, pp. 27-54, Cambridge, MA: MIT Press, 2002.

[45] M. J. Gallivan, "Striking a balance between trust and control in a virtual organization: a content analysis of open source software case studies," Information Systems Journal, vol. 11, no. 4, pp. 277-304, 2001.

[46] K. Cook, R. Hardin, and M. Levi, Cooperation Without Trust? Russell Sage Foundation Series on Trust, Vol. IX. NY: Sage, 2005.

[47] A. Brattström, D. Faems, and M. Mähring, "From trust convergence to trust divergence: Trust development in conflictual interorganizational relationships," Organization Studies, p. 0170840618789195, 2018. 